\title{
UNA EXPERIENCIA APLICADA DE METODOLOGÍA DE FLIPPED CLASSROOM: INCIDENCIA EN LA MOTIVACIÓN Y EL RENDIMIENTO EN ESTUDIANTES DE EDUCACIÓN SUPERIOR
}

An applied experience of flipped classroom: impact on higher education students' motivation and academic performance

Uma experiência aplicada de metodologia da flipped classroom: efeito sobre motivação e desempenho em estudantes do ensino superior

Plan de Apoyo a los Grupos de Innovación Docente de la ULE (PAGID 2016). Título del Proyecto financiado: "Propuesta de puesta en marcha de la metodología activa Flipped Classroom para fomentar la participación y motivación de los estudiantes de Grado"

Raquel Poy Castro (1)

Cristina Mendaña Cuervo (2)

Anselma González Fernández (3)

Ma Victoria Arana Suárez (4)

Enrique López González (5)

(1) Universidad de León, España. Teléfono: +34987295137. Correo: rpoyc@unileon.es

(2) Universidad de León, España. Teléfono: +34987291742. Correo: cristina.mendana@,unileon.es

(3) Universidad de León, España. Teléfono: +34987293025. Correo: anselma.gonzalez@unileon.es

(4) Universidad de León, España. Teléfono: +34987291467. Correo: mvaras@unileon.es

(5) Universidad de León, España. Teléfono: +34987291742. Correo: enrique.lopez@unileon.es

\begin{abstract}
Resumen
El objetivo de esta experiencia educativa fue poner en práctica una metodología activa (Flipped Classroom) con el fin de comprobar su incidencia tanto en la motivación como Una experiencia aplicada de metodología de Flipped Clasrroom: incidencia en la motivación y el rendimiento en estudiantes de Educación Superior


en los resultados académicos de los alumnos. Los alumnos de la asignatura "Teoría e Instituciones Contemporáneas de la Educación" del Grado en Educación Infantilfueron distribuidos al azar en un grupo experimental -GE- (sometido a la nueva metodología) y un grupo control -GC- al que se le aplicó la metodología tradicional. Para el contraste de hipótesis y su análisis se recurrió a técnicas estadísticas paramétricas. Los resultados evidencian una diferencia significativa entre el GE y el GC en relación a la motivación, pero no en relación al rendimiento académico. Estos resultados pueden ser explicados en base a la información obtenida durante el desarrollo de esta experiencia.

Palabras clave: Motivación; rendimiento académico; educación superior

\begin{abstract}
The aim of this study is to implement Flipped Classroom and to check the effect of this methodology on the students' motivation and academic performance. The students of subject "Contemporary Educational Theories and Institutions" of the degree in Infant Education were randomly an experimental distributed group -EG- (to which the new methodology was applied) and a control group-CG- (to which the traditional methodology was applied). To contrast the research hypotheses and their analyses, parametric statistical measurements were used. The results show a significant difference between EG and CG in relation to motivation, but not to academic performance. These results can be explained through the information obtained in this experience.
\end{abstract}

Keywords: Motivation, academic performance, higher education

\title{
Resumo
}

O objetivo desta experiência educacional foi implementar uma metodologia ativa (Flipped Classroom), a fim de verificar o seu impacto na motivação e desempenho acadêmico dos estudantes. Pupilas do "Teoria e instituições contemporâneas de Educação" sujeito Mestrado na Infância foram distribuídos aleatoriamente em um grupo experimental -GE- (submetido a nova metodologia) e um grupo de controlo ao qual aplicado o -GC- metodologia tradicional. Para os testes de hipóteses e análise, ele foi usado para paramétricos técnicas estatísticas. Os resultados mostram uma diferença significativa entre GE e GC em relação à motivação, mas não em relação ao 
desempenho acadêmico. Estes resultados podem ser explicados com base nas informações obtidas durante o curso desta experiência.

Palavras-chave: Motivação; rendimento acadêmico; Educação superior

\section{Introducción}

Desde la implantación de los estudios de Grado hemos observado un incremento en el abandono de la materia así como un empeoramiento en los resultados académicos obtenidos por los alumnos. Con el fin de paliar esta necesidad detectada, decidimos aplicar la metodología Flipped Classroom (Bergmann \& Sams, 2012) que provoque una mayor implicación/motivación de los alumnos y, probablemente, una mejora en los resultados académicos.

\section{Contextualización}

La experiencia se ha desarrollado en:

- Población: estudiantes de la Facultad de Educación de la ULE.

- Muestra de conveniencia: Alumnos de la asignatura "Teoría e Instituciones Contemporáneas de la Educación", de carácter FB, $1 \mathrm{~S}$ del $1^{\text {er }}$ curso del Grado en Educación Infantil, carga crediticia 6 ECTs.

\section{Diseño}

La experiencia educativa propuesta se enmarca dentro de un diseño experimental de investigación. Como método de evaluación se plantea dentro de los diseños experimentales un "diseño postest con grupo control” que se representa en la Tabla 1.

Tabla 1.

Diseño de la experiencia

\begin{tabular}{ccc}
\hline GE & X & O1 \\
ASIGNADO AL AZAR & TRATAMIENTO & PRUEBAS DE EVALUACIÓN \\
GC & Flipped Classroom & O2 \\
ASIGNADOS AL AZAR & & PRUEBAS DE EVALUACIÓN \\
& VI & VD \\
\hline
\end{tabular}

Para ello, se distribuyó a los estudiantes en dos grupos al azar. Al grupo experimental (GE) le aplicó la metodología Flipped Classroom (X); al grupo de control 
(GC) la metodología tradicional; al finalizar el programa se realizó a ambos grupos una prueba de evaluación ( $\mathrm{O} 1$ y $\mathrm{O} 2)$ para ver su mejora en el aprendizaje

\section{Desarrollo}

La metodología se aplicó en el primer tema de la asignatura, por agrupar los contenidos conceptuales básicos para el desarrollo y comprensión de la materia. Adicionalmente, ante la evidencia de que en los cursos anteriores los alumnos implicados no habían tenido experiencias en metodologías activas, suponía un primer acercamiento tanto a la nueva metodología como al contenido fundamental de la materia.

\section{Evaluación}

\section{Hipótesis de investigación 1. Motivación}

Con la finalidad de comprobar la motivación del alumnado, se compararon los porcentajes de alumnos (Tabla 2 ) que se presentaron a la prueba de evaluación $\left(\mathrm{P}_{\mathrm{GC}} \mathrm{y}\right.$ $\mathrm{P}_{\mathrm{GE}}$ ), asumiendo que los alumnos a los que se les aplica la nueva metodología se han sentido más motivados, o más implicados en la asignatura.

Tabla 2.

Alumnos presentados a las pruebas de evaluación en el GC y en el GE

\begin{tabular}{llcl}
\hline & $\mathbf{N}^{\mathbf{0}}$ de alumnos & Presentados & Porcentaje presentados \\
\hline $\mathrm{GE}$ & 43 matriculados & 43 & $100 \%\left(\mathrm{P}_{\mathrm{GE}}\right)$ \\
$\mathrm{GC}$ & 49 matriculados & 44 & $89,79 \%\left(\mathrm{P}_{\mathrm{GC}}\right)$ \\
\hline
\end{tabular}

Por tanto:

- $\mathrm{H}_{0}$ : La proporción de presentados es la misma en el GC y en el GE $\left(\mathrm{P}_{\mathrm{GC}}=\mathrm{P}_{\mathrm{GE}}\right)$

- $\mathrm{H}_{1}$ : La proporción de presentados no es la misma en el GC y en el GE $\left(\mathrm{P}_{\mathrm{GC}} \neq \mathrm{P}_{\mathrm{GE}}\right)$

Con los datos obtenidos, el resultado del contraste de hipótesis de diferencia de proporciones en dos muestras independientes para la materia da como valor del estadístico $Z=2,15405$ y p-valor 0,01561

Hipótesis de investigación 2. Rendimiento académico

En este caso, se trata de comprobar si las calificaciones de la prueba de evaluación realizada han supuesto una diferencia significativa entre el GE y GC. 
Con el fin de comprobar los supuestos de normalidad y homocedasticidad exigibles para la aplicación de una técnica paramétrica, se procedió a realizar las pruebas de normalidad, pudiendo asumir normalidad en ambas muestras (Tabla 3).

Tabla 3.

Pruebas de normalidad

\begin{tabular}{llr|r|r|r|r|r} 
& & \multicolumn{3}{c|}{ Kolmogorov-Smirnov } & \multicolumn{3}{c}{ Shapiro-Wilk } \\
& & Estadístico & gl & Sig. & Estadístico & gl & \multicolumn{1}{c}{ Sig. } \\
\hline "GC" Calificaciones &, 096 & 44 &, $200^{*}$ &, 963 & 44 &, 164 \\
\hline "GE" Calificaciones &, 084 & & 43 &, $200^{*}$ &, 977 & 43 &, 521 \\
\hline
\end{tabular}

*. Esto es un límite inferior de la significación verdadera.

a. Corrección de significación de Lilliefors

Por tanto, para la comparación de medias es posible realizar la prueba paramétrica t-Student, de forma que planteamos la siguiente hipótesis:

- $\mathrm{H}_{0}$ : La media de las calificaciones es la misma en el GC y en el GE

- $\mathrm{H}_{1}$ : La media de las calificaciones no es la misma en el GC y en el GE

El resultado obtenido se muestra en la Tabla 4.

\section{Tabla 4.}

Comparación de medias

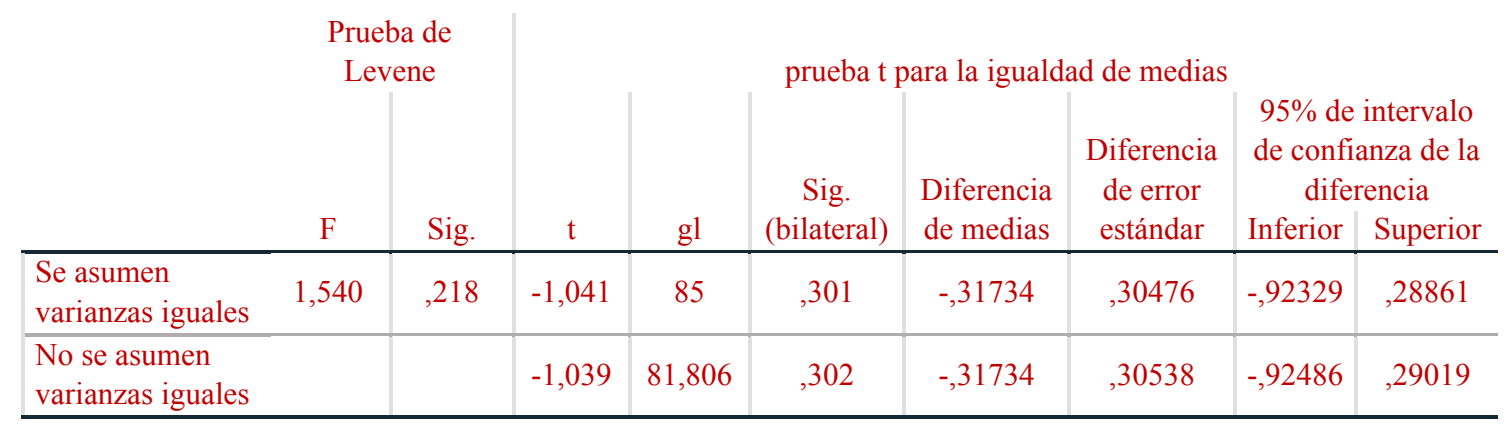

\section{Conclusiones}

\section{Hipótesis de investigación 1. Motivación}

Los resultados obtenidos, para un nivel de significación de 5\%, permiten concluir que se rechaza $\mathrm{H}_{0}$, asumiendo, por tanto, que la proporción de alumnos presentados en el GE es significativamente mayor que en el GC, lo que se puede traducir en una mayor motivación de los estudiantes.

Una experiencia aplicada de metodología de Flipped Clasrroom: incidencia en la motivación y el rendimiento en estudiantes de Educación Superior 
Hipótesis de investigación 2. Rendimiento académico

En el caso de la prueba de evaluación (Tabla 4), el valor del estadístico t es $\mathrm{t}=$ 1,041, con p-valor 0,301. El intervalo de confianza obtenido para la diferencia de medias $(-0,92329$ y 0,28861$)$ indica que se debe aceptar $\mathrm{H}_{0}$.

Esto permite aceptar que las medias son estadísticamente iguales o, lo que es lo mismo, no se han encontrado diferencias significativas entre el rendimiento académico del GC y del GE.

\section{Referencias}

Al-Zahrani, A. M. (2015).From passive to active: The impact of the flipped classroom through social learning platforms on higher education students' creative thinking. British Journal of Educational Technology, 46, 1133-1148. doi:10.1111/bjet.12353

Argente, E., García-Fornes, A., \& Espinosa, A. (2016). Aplicando la metodología Flipped-Teaching en el Grado de Ingeniería Informática: Una experiencia práctica. Actas de las XXII Jenui, Almería, 221-228. doi: http://hdl.handle.net/10251/78306

Bergmann, J., \& Sams, A. (2012). Flip your classroom: Reach every student in every class every day. Washington, DC: International Society for Technology in Education.

Caviglia-Harris, J. (2016). Flipping the Undergraduate Economics Classroom: Using Online Videos to Enhance Teaching and Learning. Southern Economic Journal, 83, 321-331. doi:10.1002/soej.12128

Cheng, X., Ka Ho Lee, K., Chang, E. Y. and Yang, X. (2016). The "flipped classroom" approach: Stimulating positive learning attitudes and improving mastery of histology among medical students. American Association of Anatomists. doi:10.1002/ase.1664

Sánchez Vera, M., Solano Fernández, I.M., \& González Calatayud, V. (2016). FLIPPEDTIC: Una experiencia de Flipped Classroom con alumnos de Magisterio. Revista Latinoamericana de Tecnología Educativa, 15(3), 69-81. doi: 10.17398/1695288X.15.3.69

Una experiencia aplicada de metodología de Flipped Clasrroom: incidencia en la motivación y el rendimiento en estudiantes de Educación Superior 